\title{
Global exponential stability for a delay differential neoclassical growth model
}

Wei Chen ${ }^{1}$ and Wentao Wang ${ }^{2^{*}}$

"Correspondence:

wwt@mail.zjxu.edu.cn

${ }^{2}$ College of Mathematics, Physics and Information Engineering,

Jiaxing University, Jiaxing, Zhejiang 314001, People's Republic of China

Full list of author information is

available at the end of the article

\begin{abstract}
This paper aims to analyze the stability of the positive equilibrium of a delay differential neoclassical growth model. We prove the existence, positivity and permanence of solutions which help to deduce the global exponential stability of the unique positive equilibrium for this model. Our method relies upon the differential inequality technique and the Lyapunov functional. Moreover, we give an example with numerical simulations to demonstrate theoretical results.
\end{abstract}

Keywords: neoclassical growth model; productivity function; delay; global exponential stability

\section{Introduction}

To explain the emergence of complex behavior even under simple economic structure, Day $[1,2]$ has seminally studied a neoclassical growth model and a productivity and population growth model which are the generalization and development of the early works of Solow [3] and Swan [4]. Tracing Day's pioneering works [1, 2], a great deal of effort in nonlinear economic dynamics was presented in Day [5], Puu [6] and Bischi et al. [7]. Due to the principle of economics, to describe the long-run behavior of the economy, the neoclassical growth model is based on the two assumptions: one is the full-employment of labor and capital and the other is instantaneous adjustment in the output market. However, it is unreasonable in real world since nonlinearities of production functions and a production delay are inevitable. Matsumoto and Szidarovszky [8] have tried to overcome the above-mentioned difficulties and have provided a special neoclassical model with a mound-shaped production function

$$
x^{\prime}(t)=s F(x(t))-\alpha x(t)
$$

where $x$ is the capital per labor, $s \in(0,1)$ is the average propensity to save and $\alpha=n+s \mu$ with $\mu$ being the depreciation ratio of capital and $n$ being the growth rate of labor; the mound-shaped production function $F(x)=A x^{a}(1-x)^{b}$ ( $a, b$ and $A$ are positive parameters) is of Cobb-Douglas type, and the factor $(1-x)^{b}$ reflects the influence of pollution on per capita output.

Following their own works, Matsumoto and Szidarovszky [9] adopt the production function $F(x)=\varepsilon x^{\gamma} e^{-\delta x}$ and consider the time delay inherent in the production process, then

\section{Springer}

(c)2014 Chen and Wang; licensee Springer. This is an Open Access article distributed under the terms of the Creative Commons Attribution License (http://creativecommons.org/licenses/by/2.0), which permits unrestricted use, distribution, and reproduction in any medium, provided the original work is properly cited. 
the delay differential neoclassical growth model is proposed as follows:

$$
x^{\prime}(t)=\beta x^{\gamma}(t-\tau) e^{-\delta x(t-\tau)}-\alpha x(t),
$$

where $\alpha, \gamma, \delta, \beta=s \varepsilon$ are positive parameters, $\tau$ is the delay in the production process, $\gamma$ can be thought of as a proxy for measuring returns to scale of the production function, $\delta$ reflects a strength of a 'negative effect' caused by increasing concentration of capital and is determined by a damaging degree of natural environment or energy resources. From the analysis of [9], when $\gamma>1$, model (1.2) has one (two) positive equilibrium(s) provided $g\left(\frac{\gamma-1}{\delta}\right)=0(>0)$, where $g(x)=-\alpha+\beta x^{\gamma-1} e^{-\delta x}$, and when $0<\gamma<1$, model (1.2) has a unique positive equilibrium. In particular, if $\gamma=1$, then model (1.2) is well-known Nicholson's blowflies model [10] and it has a unique positive equilibrium under the condition $\beta>\alpha$. Recently, there have been a lot of results on Nicholson's blowflies model, and we refer readers to [11-19] and the references cited therein for more details. However, to the best of our knowledge, there is not much work on model (1.2) when $\gamma \neq 1$. Although they have considered the local asymptotical stability of the positive equilibrium of model (1.1) and (1.2) in $[8,9]$, the results on the permanence and global stability of the delay differential neoclassical model do not appear heretofore.

Motivated by the above discussions, in this paper we mainly study the global exponential stability of the unique positive equilibrium of model $(1.2)$ with $\gamma \in(0,1)$.

At the outset of our results, we introduce two positive constants that will be crucial. Since the function $h(x)=e^{-x} x^{\gamma-1}(\gamma-x)$ is decreasing with the range $(0, \gamma+\sqrt{\gamma})$ and increasing with the range $(\gamma+\sqrt{\gamma},+\infty)$, it is easy to see that there exists a unique $\kappa \in(0, \gamma)$ such that

$$
h(\kappa)=\sup _{x \geq \kappa}|h(x)|=|h(\gamma+\sqrt{\gamma})| .
$$

Furthermore, since $x^{\gamma} e^{-x}$ increases on $[0, \gamma]$ and decreases on $[\gamma,+\infty)$, let $\widetilde{\kappa}$ be the unique number in $[\gamma,+\infty)$ such that

$$
\kappa^{\gamma} e^{-\kappa}=\widetilde{\kappa}^{\gamma} e^{-\widetilde{\kappa}}
$$

Throughout this paper, let $R^{+}$denote a nonnegative real number space, $C=C([-\tau, 0], R)$ be the Banach space of the set of all continuous functions from $[-\tau, 0]$ to $R$ equipped with supremum norm $\|\cdot\|$ and $C_{+}=C\left([-\tau, 0], R^{+}\right)$. Furthermore, for a continuous function $x$ defined on $[-\tau, \sigma)$ with $\sigma>0$, we define $x_{t} \in C$ by $x_{t}(\theta)=x(t+\theta)$ for $\theta \in[-\tau, 0]$.

It is economically reasonable to assume that only positive solutions of model (1.2) are meaningful and therefore admissible. Thus we consider admissible initial conditions and it is convenient to choose ' 0 ' as initial time for autonomous model (1.2):

$$
x_{0}=\varphi, \quad \text { where } \varphi \in C_{+} \text {and } \varphi(0)>0 .
$$

Define a continuous map $f: C_{+} \rightarrow R$ by setting

$$
f(\varphi)=\beta \varphi^{\gamma}(-\tau) e^{-\delta \varphi(-\tau)}-\alpha \varphi(0)
$$

Then $f$ is a locally Lipschitz map with respect to $\varphi \in C_{+}$, which ensures the existence and uniqueness of the solution of (1.2) with admissible initial condition (1.4). 
We write $x_{t}(\varphi)($ i.e. $x(t ; \varphi))$ for an admissible solution of admissible initial value problem (1.2) and (1.4). Also, let $[0, \eta(\varphi))$ be the maximal right interval of the existence of $x_{t}(\varphi)$.

An outline of this paper is as follows. We devote Section 2 to some lemmas and definitions on the existence, positivity and permanence of solutions, which play an important role in Section 3 to establish the global exponential stability of the positive equilibrium. In Section 4, an example and its numerical simulations are provided to illustrate our results obtained in the previous sections. The last section concludes the paper.

\section{Preliminary results}

In this section, we present some lemmas and definitions to prove our main results in Section 3.

Definition 2.1 Let $\bar{x}$ be the unique positive equilibrium of model (1.2). If there exist constants $\lambda>0, \widetilde{K}>0$ and $T>0$ such that every solution $x(t ; \varphi)$ to the initial value problem (1.2) and (1.4) always satisfies

$$
|x(t ; \varphi)-\bar{x}| \leq \widetilde{K} e^{-\lambda t} \quad \text { for all } t>T,
$$

then $\bar{x}$ is said to be globally exponentially stable.

Definition 2.2 All solutions are uniformly permanent if there exist positive constants $m$ and $M$ such that for any solution $x(t)$, we have

$$
m<\liminf _{t \rightarrow \infty} x(t) \leq \limsup _{t \rightarrow \infty} x(t)<M
$$

Lemma 2.1 There exists a unique positive global solution of model (1.2) and (1.4) on the interval $[0,+\infty)$.

Proof Because of $\varphi \in C_{+}$, we have $x_{t}(\varphi) \in C_{+}$by using Theorem 5.2.1 in [20]. Set $x(t)=$ $x(t ; \varphi)$ for $t \in[0, \eta(\varphi))$. From the variation of constants formula and initial condition $\varphi(0)>$ 0 , we obtain

$$
x(t)=\varphi(0) e^{-\alpha t}+\beta \int_{0}^{t} e^{-\alpha(t-s)} x^{\gamma}(s-\tau) e^{-\delta x(s-\tau)} d s>0
$$

for all $t \in[0, \eta(\varphi))$.

It remains to show $\eta(\varphi)=+\infty$.

For the sake of contradiction, assume that $\eta(\varphi)$ is bounded. Note the fact that $\sup _{x \in[0,+\infty)} x^{\gamma} e^{-x}=\gamma^{\gamma} e^{-\gamma}$, we obtain from (1.2) that

$$
\begin{aligned}
x^{\prime}(t) & =\frac{\beta}{\delta^{\gamma}}(\delta x(t-\tau))^{\gamma} e^{-\delta x(t-\tau)}-\alpha x(t) \\
& \leq \frac{\beta \gamma^{\gamma}}{\delta^{\gamma} e^{\gamma}}-\alpha x(t), \quad t \geq 0 .
\end{aligned}
$$

This leads to

$$
x(t) \leq \varphi(0) e^{-\alpha t}+\frac{\beta \gamma^{\gamma}}{\delta^{\gamma} e^{\gamma} \alpha}\left(1-e^{-\alpha t}\right), \quad t \geq 0,
$$


which excludes the possibility that $\lim _{t \rightarrow \eta(\varphi)^{-}} x(t)=\infty$. Hence it violates Theorem 2.3.1 in [21]. So we obtain the existence of the unique global positive solution of (1.2) and (1.4) on $[0,+\infty)$. Therefore Lemma 2.1 is proved.

Lemma 2.2 Suppose that there exists a positive constant $K \in(\kappa, \widetilde{\kappa})$ such that

$$
\frac{\beta \gamma^{\gamma} \delta^{1-\gamma}}{K e^{\gamma}}<\alpha<\frac{\beta \delta^{1-\gamma}}{e^{\kappa}}
$$

then solutions of (1.2) and (1.4) are uniformly permanent with

$$
m=\frac{\kappa}{\delta}, \quad M=\frac{K}{\delta} .
$$

Proof Let $x(t)=x(t ; \varphi)$. By Lemma 2.1, $x(t)>0$ for $t \geq 0$. From Theorem 1.6.1 in [22] and (2.1), we get that the solution $x(t)$ to the initial value problem (1.2) and (1.4) is not greater than the solution to the initial value problem

$$
y^{\prime}(t)=\frac{\beta \gamma^{\gamma}}{\delta^{\gamma} e^{\gamma}}-\alpha y(t), \quad t \geq 0, \quad y(0)=x(0) .
$$

In view of $(2.2)$ and

$$
y(t)=x(0) e^{-\alpha t}+\frac{\beta \gamma^{\gamma}}{\delta^{\gamma} e^{\gamma} \alpha}\left(1-e^{-\alpha t}\right),
$$

we have

$$
\limsup _{t \rightarrow \infty} x(t) \leq \limsup _{t \rightarrow \infty} y(t)=\frac{\beta \gamma^{\gamma}}{\delta^{\gamma} e^{\gamma} \alpha}<\frac{K}{\delta} .
$$

We next show that $l=\liminf _{t \rightarrow \infty} x(t)>0$. Otherwise, we assume that $l=0$. For each $t \geq 0$, we define

$$
m(t)=\max \left\{\xi \mid \xi \leq t, x(\xi)=\min _{0 \leq s \leq t} x(s)\right\} .
$$

It follows from $l=0$ that $m(t) \rightarrow \infty$ as $t \rightarrow \infty$ and that

$$
\lim _{t \rightarrow \infty} x(m(t))=0 .
$$

From the definition of $m(t)$, we know that $x^{\prime}(m(t)) \leq 0$ or

$$
\alpha x(m(t)) \geq \beta x^{\gamma}(m(t)-\tau) e^{-\delta x(m(t)-\tau)} .
$$

Then

$$
\begin{aligned}
0 & =\lim _{t \rightarrow \infty} \alpha x(m(t)) \\
& \geq \lim _{t \rightarrow \infty} \beta x^{\gamma}(m(t)-\tau) e^{-\delta x(m(t)-\tau)} \\
& \geq \lim _{t \rightarrow \infty} \beta x^{\gamma}(m(t)-\tau) e^{-K} \\
& \geq 0,
\end{aligned}
$$


which implies that

$$
\lim _{t \rightarrow \infty} x(m(t)-\tau)=0 .
$$

This yields that there exists $t_{1}>0$ such that

$$
0<\delta x(m(t)-\tau)<\kappa, \quad t \geq t_{1} .
$$

In view of (2.3), (2.4) and $\kappa, \gamma \in(0,1)$, for $t \geq t_{1}$, we have

$$
\begin{aligned}
\alpha & \geq \beta \delta^{1-\gamma} \lim _{t \rightarrow \infty} \frac{(\delta x(m(t)-\tau))^{\gamma}}{\delta x(m(t))} e^{-\delta x(m(t)-\tau)} \\
& \geq \beta \delta^{1-\gamma} \lim _{t \rightarrow \infty} \frac{\delta x(m(t)-\tau)}{\delta x(m(t))} e^{-\kappa} \\
& \geq \frac{\beta \delta^{1-\gamma}}{e^{\kappa}}
\end{aligned}
$$

which obviously contradicts with (2.2). Thus, we have proved that $l>0$.

Finally, we prove that $l>\frac{\kappa}{\delta}$. Again, by way of contradiction, we assume that $l \leq \frac{\kappa}{\delta}$. By the fluctuation lemma [22, Lemma A.1], there exists a sequence $\left\{t_{k}\right\}_{k \geq 1}$ such that

$$
t_{k} \rightarrow \infty, \quad x\left(t_{k}\right) \rightarrow \liminf _{t \rightarrow \infty} x(t), \quad x^{\prime}\left(t_{k}\right) \rightarrow 0 \quad \text { as } k \rightarrow \infty .
$$

Since $\left\{x_{t_{k}}\right\}$ is bounded and equicontinuous, by the Ascoli-Arzela theorem, there exists a subsequence, still denoted by itself for simplicity of notation, such that

$$
x_{t_{k}} \rightarrow \widetilde{\varphi} \text { for some } \widetilde{\varphi} \in C_{+} .
$$

Moreover,

$$
l=\widetilde{\varphi}(0) \leq \widetilde{\varphi}(\theta) \leq \frac{K}{\delta}<\frac{\widetilde{\kappa}}{\delta} \quad \text { for } \theta \in[-\tau, 0) .
$$

Then recall that $\delta l \leq \kappa<\gamma<1$, and it follows from

$$
x^{\prime}\left(t_{k}\right)=\beta x^{\gamma}\left(t_{k}-\tau\right) e^{-\delta x\left(t_{k}-\tau\right)}-\alpha x\left(t_{k}\right)
$$

that (taking limits)

$$
\begin{aligned}
0 & =\frac{\beta}{\delta^{\gamma}}(\delta \widetilde{\varphi}(-\tau))^{\gamma} e^{-\delta \widetilde{\varphi}(-\tau)}-\alpha l \\
& \geq \frac{\beta}{\delta^{\gamma}}(\delta l)^{\gamma} e^{-\delta l}-\alpha l \\
& \geq l\left(\beta \delta^{1-\gamma}(\delta l)^{\gamma-1} e^{-\kappa}-\alpha\right) \\
& >l\left(\frac{\beta \delta^{1-\gamma}}{e^{\kappa}}-\alpha\right)
\end{aligned}
$$

which contradicts with (2.2). This proves that $l>\frac{\kappa}{\delta}$. Hence the proof of Lemma 2.2 is completed. 


\section{Main results}

In this section, we establish sufficient conditions on the global exponential stability for the unique positive equilibrium of model (1.2).

Theorem 3.1 Suppose that all conditions in Lemma 2.2 are satisfied and assume that

$$
\alpha>\frac{\beta}{\delta^{\gamma}}|h(\gamma+\sqrt{\gamma})| .
$$

Then the unique positive equilibrium $\bar{x}$ of model (1.2) is globally exponentially stable, that is, there exist constants $\lambda>0, \widetilde{K}>0$ and $T>0$ such that

$$
|x(t ; \varphi)-\bar{x}|<\widetilde{K} e^{-\lambda t} \quad \text { for all } t>T .
$$

Proof Define a continuous function $\Gamma(u)$ as

$$
\Gamma(u)=u-\alpha+\frac{\beta}{\delta^{\gamma}}|h(\gamma+\sqrt{\gamma})| e^{u \tau}, \quad u \in[0,1] .
$$

From (3.1), we obtain

$$
\Gamma(0)=-\alpha+\frac{\beta}{\delta^{\gamma}}|h(\gamma+\sqrt{\gamma})|<0,
$$

which implies that there exist two constants $\eta>0$ and $\lambda \in(0,1]$ such that

$$
\Gamma(\lambda)=\lambda-\alpha+\frac{\beta}{\delta^{\gamma}}|h(\gamma+\sqrt{\gamma})| e^{\lambda \tau}<-\eta<0 .
$$

For convenience of notation, denote $x(t ; \varphi)$ by $x(t)$. Lemma 2.2 implies that there exists $T>0$ such that

$$
\frac{\kappa}{\delta}<x(t)<\frac{K}{\delta}<\frac{\widetilde{\kappa}}{\delta} \quad \text { for all } t \in[T-\tau,+\infty) .
$$

Moreover, since the positive equilibrium $\bar{x}$ is the special solution of model (1.2), Lemma 2.2 also implies that

$$
\frac{\kappa}{\delta}<\bar{x}<\frac{K}{\delta}<\frac{\widetilde{\kappa}}{\delta} .
$$

Set $y(t)=x(t)-\bar{x}, t \in[-\tau,+\infty)$. For $t \geq 0$, we have

$$
y^{\prime}(t)=\beta\left(x^{\gamma}(t-\tau) e^{-\delta x(t-\tau)}-\bar{x}^{\gamma} e^{-\delta \bar{x}}\right)-\alpha y(t) .
$$

Next we consider the Lyapunov functional

$$
V(t)=|y(t)| e^{\lambda t}
$$

For all $t>T$, calculating the upper left derivative of $V(t)$ along with the solution $y(t)$ of (3.5), we have

$$
D^{-}(V(t)) \leq(\lambda-\alpha)|y(t)| e^{\lambda t}+\beta\left|x^{\gamma}(t-\tau) e^{-\delta x(t-\tau)}-\bar{x}^{\gamma} e^{-\delta \bar{x}}\right| e^{\lambda t} .
$$


Now, we claim that

$$
\begin{aligned}
V(t) & =|y(t)| e^{\lambda t} \\
& <e^{\lambda T}\left(\max _{t \in[-\tau, T]}|x(t)-\bar{x}|+1\right) \\
& :=\widetilde{K} \quad \text { for all } t>T .
\end{aligned}
$$

If this is not true, then there must exist $s_{1}>T$ such that

$$
V\left(s_{1}\right)=\widetilde{K} \quad \text { and } \quad V(t)<\widetilde{K} \quad \text { for all } t \in\left[-\tau^{+}, s_{1}\right)
$$

Then (3.2), (3.3), (3.4) and (3.7) yield

$$
\begin{aligned}
0 & \leq D^{-}\left(V\left(s_{1}\right)\right) \\
& \leq(\lambda-\alpha)\left|y\left(s_{1}\right)\right| e^{\lambda s_{1}}+\frac{\beta}{\delta \gamma}\left|\left(\delta x\left(s_{1}-\tau\right)\right)^{\gamma} e^{-\delta x\left(s_{1}-\tau\right)}-(\delta \bar{x})^{\gamma} e^{-\delta \bar{x}}\right| e^{\lambda s_{1}} \\
& \leq(\lambda-\alpha) V\left(s_{1}\right)+\frac{\beta}{\delta^{\gamma}}|h(\gamma+\sqrt{\gamma})| e^{\lambda \tau} V\left(s_{1}-\tau\right) \\
& <\widetilde{K}\left[(\lambda-\alpha)+\frac{\beta}{\delta \gamma}|h(\gamma+\sqrt{\gamma})| e^{\lambda \tau}\right] \\
& <-\widetilde{K} \eta \\
& <0
\end{aligned}
$$

which is a contradiction. Here we have applied the inequality

$$
\left|x_{1}^{\gamma} e^{-\delta x_{1}}-x_{2}^{\gamma} e^{-\delta x_{2}}\right| \leq|h(\gamma+\sqrt{\gamma})|\left|x_{1}-x_{2}\right| \quad \text { for } x_{1}, x_{2} \in[\kappa,+\infty),
$$

which can be easily obtained by (1.3) and the differential mean value theorem. Hence, (3.8) holds and

$$
|x(t)-\bar{x}|=|y(t)|<\widetilde{K} e^{-\lambda t} \quad \text { for all } t>T .
$$

This completes the proof of Theorem 3.1.

\section{An example}

In this section, we give an example and its numerical simulations in order to support the results obtained in previous sections.

Example 4.1 Consider the following delay differential neoclassical growth model:

$$
x^{\prime}(t)=4 x^{0.5}(t-1) e^{-x(t-1)}-2.8 x(t), \quad t \geq 0 .
$$

Obviously, $\alpha=2.8, \beta=4, \gamma=0.5, \delta, \tau=1$. 


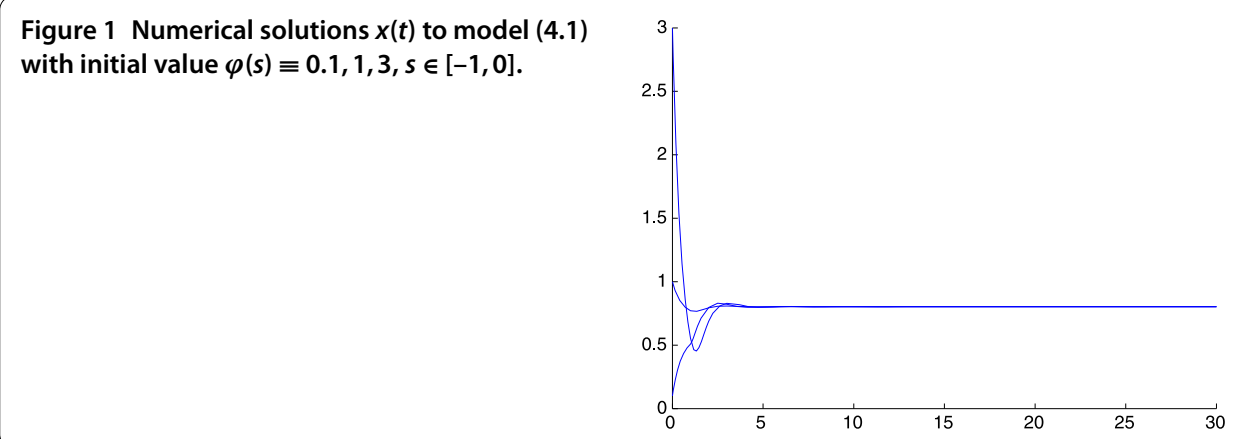

Note that $\kappa \approx 0.341671$ and $\widetilde{\kappa} \approx 0.701012$. Let $K=0.7 \in(\kappa, \widetilde{\kappa})$. Then

$$
\begin{aligned}
& 2.45074 \approx \frac{\beta \gamma^{\gamma} \delta^{1-\gamma}}{K e^{\gamma}}<\alpha<\frac{\beta \delta^{1-\gamma}}{e^{\kappa}} \approx 2.84233, \\
& 2.8=\alpha>\frac{\beta}{\delta^{\gamma}}|h(\gamma+\sqrt{\gamma})| \approx 0.769896 .
\end{aligned}
$$

Hence, from Theorem 3.1, the unique positive equilibrium $\bar{x} \approx 0.802906$ of model (4.1) is globally exponentially stable with exponential convergence rate $\lambda \approx 0.5$. The numerical simulations in Figure 1 strongly support this conclusion.

Remark 4.1 To the best of our knowledge, few authors have considered the global exponential stability of the unique positive equilibrium for model (1.2). It is clear that all the results in $[8,9]$ and the references therein cannot be applicable to prove that all solutions to model (4.1) with initial value (1.4) converge exponentially to the positive equilibrium. So the results of this paper are essentially new and complement the work of Matsumoto and Szidarovszky $[8,9]$.

\section{Conclusion}

In the paper, we have studied a special delay differential neoclassical model. A moundshaped production for capital growth and the delay in the production process are assumed in the dynamic equation. We have established some criteria to ensure the global exponential stability of the unique positive equilibrium for model $(1.2)$ with $\gamma \in(0,1)$. In the future, we will investigate model (1.2) with $\gamma>1$ since it has more complex dynamic behavior and there possibly exist two positive equilibria.

Competing interests

The authors declare that they have no competing interests.

Authors' contributions

The authors declare that the study was realized in collaboration with the same responsibility. Both authors read and approved the final version of the manuscript.

\section{Author details}

${ }^{1}$ School of Mathematics and Information, Shanghai Lixin University of Commerce, Shanghai, 201620, People's Republic of China. ${ }^{2}$ College of Mathematics, Physics and Information Engineering, Jiaxing University, Jiaxing, Zhejiang 314001 , People's Republic of China.

\section{Acknowledgements}

This work was supported by the National Natural Science Foundation of China (Grant Nos. 11301341, 11201184) and Innovation Program of Shanghai Municipal Education Commission (Grant No. 13YZ127). 


\section{References}

1. Day, R: Irregular growth cycles. Am. Econ. Rev. 72, 406-414 (1982)

2. Day, R: The emergence of chaos from classical economic growth. Q. J. Econ. 98, 203-213 (1983)

3. Solow, R: A contribution to the theory of economic growth. Q. J. Econ. 70, 65-94 (1956)

4. Swan, T: Economic growth and capital accumulation. Econ. Rec. 32, 334-361 (1956)

5. Day, R: Complex Economic Dynamics: An Introduction to Dynamical Systems and Market Mechanism. MIT Press, Cambridge (1994)

6. Puu, T: Attractions, Bifurcations and Chaos: Nonlinear Phenomena in Economics, 2nd edn. Springer, Berlin (2003)

7. Bischi, Gl, Chiarella, C, Kopel, M, Szidarovszky, F: Nonlinear Oligopolies: Stability and Bifurcation. Springer, Berlin (2010)

8. Matsumoto, A, Szidarovszky, F: Delay differential neoclassical growth model. J. Econ. Behav. Organ. 78, 272-289 (2011)

9. Matsumoto, A, Szidarovszky, F: Asymptotic behavior of a delay differential neoclassical growth model. Sustainability 5, 440-455 (2013)

10. Gurney, W, Blythe, S, Nisbet, R: Nicholson's blowflies revisited. Nature 287, 17-21 (1980)

11. Chen, Y: Periodic solutions of delayed periodic Nicholson's blowflies models. Can. Appl. Math. Q. 11, $23-28$ (2003)

12. Berezansky, L, Braverman, E, Idels, L: Nicholson's blowflies differential equations revisited: main results and open problems. Appl. Math. Model. 34, 1405-1417 (2010)

13. Chen, W, Liu, B: Positive almost periodic solution for a class of Nicholson's blowflies model with multiple time-varying delays. J. Comput. Appl. Math. 235, 2090-2097 (2011)

14. Wang, W, Wang, L, Chen, W: Existence and exponential stability of positive almost periodic solution for Nicholson-type delay systems. Nonlinear Anal., Real World Appl. 12, 1938-1949 (2011)

15. Wang, W: Positive periodic solutions of delayed Nicholson's blowflies models with a nonlinear density-dependent mortality term. Appl. Math. Model. 36, 4708-4713 (2012)

16. Wang, L: Almost periodic solution for Nicholson's blowflies model with patch structure and linear harvesting terms Appl. Math. Model. 37, 2153-2165 (2013)

17. Yi, T, Zou, X: Global attractivity of the diffusive Nicholson blowflies equation with Neumann boundary condition: a non-monotone case. J. Differ. Equ. 245, 3376-3388 (2008)

18. Shu, H, Wang, L, Wu, J: Global dynamics of Nicholson's blowflies equation revisited: onset and termination of nonlinear oscillations. J. Differ. Equ. 255, 2565-2586 (2013)

19. Liu, B: Global exponential stability of positive periodic solutions for a delayed Nicholson's blowflies model. J. Math Anal. Appl. 412, 212-221 (2014)

20. Smith, HL: Monotone Dynamical Systems. Math. Surveys Monogr. Am. Math. Soc., Providence (1995)

21. Hale, J, Verduyn Lunel, S: Introduction to Functional Differential Equations. Applied Mathematical Sciences, vol. 99 Springer, New York (1993)

22. Hale, J: Ordinary Differential Equations. Wiley-Interscience, New York (1980)

10.1186/1687-1847-2014-325

Cite this article as: Chen and Wang: Global exponential stability for a delay differential neoclassical growth model. Advances in Difference Equations 2014, 2014:325

\section{Submit your manuscript to a SpringerOpen ${ }^{\circ}$ journal and benefit from:}

- Convenient online submission

- Rigorous peer review

- Immediate publication on acceptance

- Open access: articles freely available online

- High visibility within the field

- Retaining the copyright to your article 